\title{
Nonlinear response with dichotomous noise
}

\author{
I. Bena and C. Van den Broeck \\ Limburgs Universitair Centrum, B-3590 Diepenbeek, Belgium \\ R. Kawai \\ Department of Physics, University of Alabama at Birmingham, Birmingham, Alabama 35294 \\ Katja Lindenberg \\ Department of Chemistry and Biochemistry 0340 and Institute for Nonlinear Science, University of California, San Diego, \\ La Jolla, California 92093 \\ (Received 15 July 2002; published 21 October 2002)
}

\begin{abstract}
Dichotomous noise appears in a wide variety of physical and mathematical models. It has escaped attention that the standard results for the long time properties cannot be applied when unstable fixed points are crossed in the asymptotic regime. We show how calculations have to be modified to deal with these cases and present as a first application full analytic results for hypersensitive transport.
\end{abstract}

DOI: 10.1103/PhysRevE.66.045603

PACS number(s): 43.50.+y, 05.40.-a, 02.50.-r

While the Wiener process together with its "time derivative," the Gaussian white noise, is certainly the method of choice to describe Brownian motion, the motion induced by another fundamental stochastic process, namely, the dichotomous Markov process [see, e.g., Refs. [1,2]], has its own virtues and interest. Systems driven by dichotomous noise can often be described analytically, including the Gaussian white noise case as a specific limit, and allow the analytic investigation of the effects of the finite correlation time of the noise, notably in noise induced transitions, noise induced phase transitions, stochastic resonance, and ratchets. Dichotomous noise is often a good representation of the actual physical situation, e.g., thermal transitions between two configurations or states, and can easily be implemented as an external noise, with the additional advantage that the support of this noise is finite.

A widely studied generic stochastic equation that describes the temporal evolution of a single scalar variable $x(t)$ is

$$
\dot{x}=\xi(t) v(x)+F
$$

where the dot stands for the time derivative, $\xi(t)$ is a symmetric dichotomous Markov process that takes on the values \pm 1 with transition rate $k, v(x)$ is a given velocity profile, and $F$ is a constant external force. One can of course embellish this description in a variety of ways such as, e.g., by allowing for a state- and/or time-dependent external force, but here we adhere to this simple form. Existing results include the steady state distribution $[3,4]$ and first passage time moments, see, e.g., Ref. [5]. When Eq. (1) is defined on an interval with periodic boundary conditions, one can evaluate the stationary probability flux and from it the average asymptotic drift velocity or diffusion coefficient, a problem that has recently received a great deal of attention in the context of Josephson junctions and Brownian motors [6]. Although these results are often claimed to be completely general, our study shows that this is not the case. Indeed, to our knowledge, with the exception of Ref. [7], all the exist- ing results are limited to motion that asymptotically does not cross unstable fixed points of the dynamics. The main purpose of our work is to point out where the existing results break down, and to present the procedure to obtain a fully general solution for the asymptotic average velocity, including, as a first direct application, the problem of hypersensitive response [8].

Consider, then, the prototypical stochastic differential equation (1). We take the velocity profile $v(x)$ to be periodic, $v(x)=v(x+L)$, with zero average, $\int_{0}^{L} v(x) d x=0$. A schematic representation of the two configurations assumed by the "net potentials" $\mp V(x)-F x$ associated with the right hand side [with $v(x) \equiv-d V(x) / d x$ ] is shown in Fig. 1. The fixed points of the dynamics are the points at which $\pm v(x)$ $+F$ vanish, i.e., the local extrema of the net potentials. The stochastic dynamics (1) can equivalently be described by the master equation for the probability densities $P_{+}(x, t)$ and $P_{-}(x, t)$ for being at $x$ at time $t$ if $\xi=+1$ and -1 , respectively, with $x \in[0, L]$ and periodic boundary conditions:

$$
\begin{aligned}
\frac{\partial P_{ \pm}(x, t)}{\partial t}= & \mp \frac{\partial}{\partial x}\left\{[v(x) \pm F] P_{ \pm}(x, t)\right\} \\
& -k\left[P_{ \pm}(x, t)-P_{\mp}(x, t)\right] .
\end{aligned}
$$

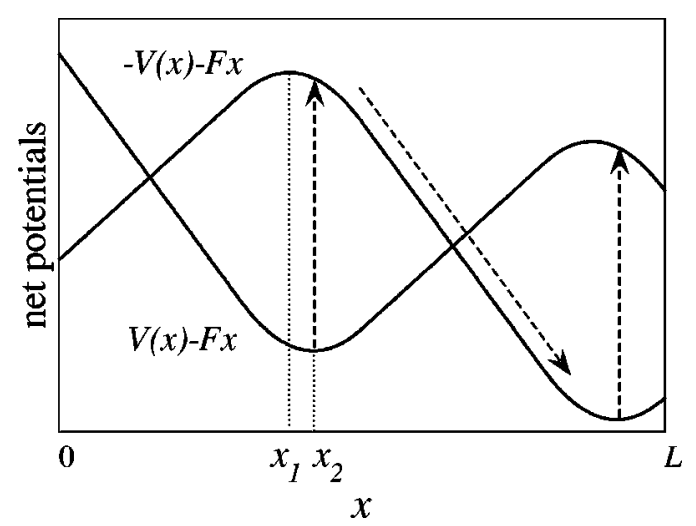

FIG. 1. The net potentials $\mp V(x)-F x$. 
To find analytic expressions for the asymptotic steady state (i.e., time-independent) probabilities and mean velocity $\langle\dot{x}\rangle$, it is more convenient to introduce the sum and difference probability densities $P(x, t)=P_{+}(x, t)+P_{-}(x, t)$ and $p(x, t)=P_{+}(x, t)-P_{-}(x, t)$. Summation of the equations in Eq. (2) then immediately leads to the conclusion that in the steady state the probability flux $J$ associated with $P(x)$, namely, $J=F P(x)+v(x) p(x)$, is a constant whose value is to be determined. It also leads to a direct relation between the mean velocity in the stationary state and the flux, $\langle\dot{x}\rangle$ $=\int_{0}^{L}\left\{[v(x)+F] P_{+}(x)+[-v(x)+F] P_{-}(x)\right\} d x=L J$.

Again in the steady state, subtraction of the equations in Eq. (2) and the constant flux condition leads to the following first-order differential equation for $p(x)$ :

$$
\left[F^{2}-v^{2}(x)\right] \frac{d p(x)}{d x}-2\left[v(x) v^{\prime}(x)-k F\right] p(x)+J v^{\prime}(x)=0,
$$

where $v^{\prime}(x)=d v(x) / d x$. The solution to this equation, together with the constant flux condition and the normalization condition for $P(x), \int_{0}^{L} P(x) d x=1$, can be used to determine $P(x)$, the flux $J$ and the mean velocity $\langle\dot{x}\rangle$.

The crux of the problem now resides in finding the solution to Eq. (3). This solution is straightforward when $\left[F^{2}\right.$ $\left.-v^{2}(x)\right]$ has no zeroes, that is, when the net potentials have no extrema within the interval $(0, L)$. In this case the standard method of variation of parameters leads to the familiar solution

$$
p(x)=-\frac{J}{\left|F^{2}-v^{2}(x)\right|}[C G(x, 0)+H(x, 0 ; x)],
$$

where $C$ is a constant of integration that arises from the general solution to the homogeneous part of Eq. (3), the second contribution is the particular solution, and we have defined the functions

$$
\begin{gathered}
H(z, y ; x)=\int_{y}^{z} \operatorname{sgn}\left[F^{2}-v^{2}\left(x^{\prime}\right)\right] v^{\prime}\left(x^{\prime}\right) G\left(x, x^{\prime}\right) d x^{\prime}, \\
G(z, y) \equiv \exp \left[-2 k F \int_{y}^{z} \frac{d x}{F^{2}-v^{2}(x)}\right] .
\end{gathered}
$$

The usual procedure to determine $C$ is to require periodicity of $p(x)$, recalling that $v(x)$ is periodic. One finally obtains $p(x)=[J-F P(x)] / J v(x)$, and

$$
\begin{aligned}
P(x)= & \frac{J}{F}\left\{1-\frac{v(x)}{[1-G(0, L)]\left[F^{2}-v^{2}(x)\right]}\right. \\
& \left.\times \int_{x}^{x+L} d x^{\prime} v^{\prime}\left(x^{\prime}\right) G\left(x, x^{\prime}\right)\right\} .
\end{aligned}
$$

The normalization of $P(x)$ determines the value of the flux $J$ and leads to the following result for the mean velocity in the steady state:

$$
\begin{aligned}
\langle\dot{x}\rangle= & F\left\{1-\frac{1}{L[1-G(0, L)]} \int_{0}^{L} d x \frac{v(x)}{F^{2}-v^{2}(x)}\right. \\
& \left.\times \int_{x}^{x+L} d x^{\prime} v^{\prime}\left(x^{\prime}\right) G\left(x, x^{\prime}\right)\right\}^{-1} .
\end{aligned}
$$

The standard results shown above are applicable not only in the absence of fixed points, but also when the asymptotic behavior is governed by stable fixed points. In this latter case the dynamics settles into an alternating motion between these points, so that they delimit the interval in which the steady state probability is nonzero [4]. The associated normalizable divergences at the fixed points represent regions where the probability density for finding the system is high.

The situation is entirely different, both physically and mathematically, when the system can cross unstable fixed points within the interval $(0, L)$ in the long time limit. A simple illustration is provided by the example $v(x)=\sin x$. In the absence of an external force, the dynamics is restricted to an interval $[k \pi,(k+1) \pi]$ ( $k$ integer). Even though the application of an external forcing $|F|<1$ cannot induce "escape" from this interval in either of the separate dynamics $\dot{x}=\sin x+F$ and $\dot{x}=-\sin x+F$, running solutions with finite average velocity appear when the dynamics switches back and forth between the two [8] (see Fig. 1). The explicit calculation of this velocity is one of our main goals. Clearly, the solution (6) is no longer correct because it contains nonintegrable singularities (see below) at the unstable fixed points where the probability of finding the system is expected to be low, not high.

For simplicity we restrict our presentation to velocity profiles $v(x)$ that are continuously decreasing functions of $x$ on $[0, L / 2]$ and symmetric about $L / 2, v(x+L / 2)=-v(x)$. This implies that $P(x+L / 2)=P(x)$ and $p(x+L / 2)=-p(x)$, so that we can limit our analysis to the interval $[0, L / 2]$. In this "minimal scenario," the equation $F^{2}-v^{2}(x)=0$ has only two solutions in the interval $[0, L / 2]$, namely, $x_{1}$, corresponding to an unstable fixed point in the $\xi=-1$ dynamics $\left[F=v\left(x_{1}\right)\right]$, and $x_{2}$, a stable fixed point in the $\xi=+1 \mathrm{dy}-$ namics $\left[F=-v\left(x_{2}\right)\right]$, with $x_{2}>x_{1}$. The steady state results leading to Eq. (3) still apply, but the solution to Eq. (3) is more delicate than the "blind" integration that yields Eq. (6). Indeed, the coefficient of the first derivative is zero at the fixed points, which now lie entirely within the support of the probability distribution. Thus, the equation becomes singular.

The method of variation of parameters for an equation of the type (3) leads to a solution which is a sum of the general solution of the homogeneous equation and a particular solution of the inhomogeneous equation, as in Eq. (4). The subtlety lies in the determination of the constant of integration $C$, which in the previous case was fixed simply by imposing periodic boundary conditions. In the vicinity of the stable fixed point $x_{2}$ this straightforward procedure leads to the dependence $P(x) \sim\left|x-x_{2}\right|^{k /\left|v^{\prime}\left(x_{2}\right)\right|-1}$, which is continuous when $k /\left|v^{\prime}\left(x_{2}\right)\right|>1$ and divergent but integrable for $k /\left|v^{\prime}\left(x_{2}\right)\right| \leqslant 1$. This result causes no mathematical difficulty and is consistent with the physical intuition that probability 
near a stable fixed point should indeed build up, especially when the switching rate is low. At the unstable fixed point $x_{1}$, however, this procedure leads to an apparent nonintegrable divergence, $P(x) \sim\left|x-x_{1}\right|^{-k /\left|v^{\prime}\left(x_{1}\right)\right|-1}$, which is clearly unphysical and mathematically improper in view of the requirement of normalization. The fallacy lies in the assumption that a single constant $C$ is valid throughout the region $(0, L)$. In fact, the solution (4) is valid in the separate intervals $\left[0, x_{1}\right),\left(x_{1}, x_{2}\right)$, and $\left(x_{2}, L / 2\right]$, but not necessarily with the same constant of integration in all of them. Indeed, there is exactly one choice of this constant valid for both $\left[0, x_{1}\right)$ and $\left(x_{1}, x_{2}\right)$ such that the divergence at $x_{1}$ is removed, and another choice valid in the interval $\left(x_{2}, L / 2\right]$ that ensures required continuity and periodicity. In other words, even though the general solution of the homogeneous equation always has a divergence, there exists exactly one solution to the full inhomogeneous equation (3) that has no divergence and is actually completely smooth at $x_{1}$. This solution is given by Eq. (4) with the choice $C=-H\left(x_{1}, 0 ; 0\right)$ in both intervals $\left[0, x_{1}\right)$ and $\left(x_{1}, x_{2}\right)$ [9]. This choice insures that the coefficient of the divergent term vanishes at $x=x_{1}$. We conclude that for $x \in\left[0, x_{2}\right)$,

$$
P(x)=\frac{J}{F}\left[1+\frac{v(x)}{\left|F^{2}-v^{2}(x)\right|} H\left(x, x_{1} ; x\right)\right] .
$$

Note that $P(x)$ is now continuous at $x_{1}$, and that $\lim _{x \backslash x_{1}} P(x)=\lim _{x \succ x_{1}} P(x)=J F^{-1}\left\{1-\left[2\left(k /\left|v^{\prime}\left(x_{1}\right)\right|\right.\right.\right.$ $\left.+1)]^{-1}\right\}$ is indeed finite.

For $x \in\left(x_{2}, L / 2\right]$, the result (4) for $p(x)$ applies again, but now the constant $C$ is determined by imposing the continuity of $p(x)$ at $x=L / 2$. One finds

$$
\begin{aligned}
P(x)= & \frac{J}{F}\left\{1+\frac{v(x)}{\left|F^{2}-v^{2}(x)\right|}[H(x, L / 2 ; x)\right. \\
& \left.\left.+G(0, L / 2) H\left(x_{1}, 0 ; x\right)\right]\right\} .
\end{aligned}
$$

At the stable fixed point $x_{2}, P(x)$ has the behavior described earlier, i.e., it is continuous for $k /\left|v_{2}^{\prime}\left(x_{2}\right)\right|>1$ and divergent but integrable for $k /\left|v^{\prime}\left(x_{2}\right)\right| \leqslant 1$.

The values of the flux $J$ and of the average velocity follow from these results by imposing the normalization of $P(x)$ :

$$
\begin{aligned}
\langle\dot{x}\rangle= & F\left\{1+\frac{2}{L} \int_{0}^{x_{2}} d x \frac{v(x)}{\left|F^{2}-v^{2}(x)\right|} H\left(x, x_{1} ; x\right)\right. \\
& +\frac{2}{L} \int_{x_{2}}^{L / 2} d x \frac{v(x)}{\left|F^{2}-v^{2}(x)\right|}[H(x, L / 2 ; x) \\
& +G(0, L / 2) H(x, 0 ; x)]\}^{-1} .
\end{aligned}
$$

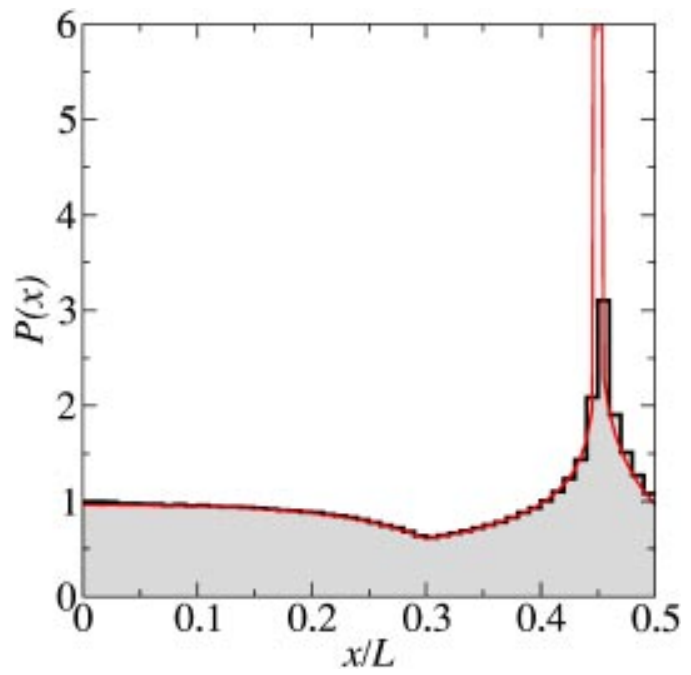

FIG. 2. Probability density $P(x)$ vs $x / L$ for the parameter values $f=0.5, \Gamma=0.4$, and $\alpha=1.0$. Histogram: simulation results. Curve: exact theory.

This is our main new result. Note that the above procedure can be repeated straightforwardly but tediously for more complicated cases involving several stable and unstable fixed points.

To illustrate our findings with explicit results, we turn to a particular case of a piecewise linear profile

$$
v(x)=\left\{\begin{array}{l}
v_{0} \text { for } x \in[0, L / 2-2 l), \\
v_{0}\left(\frac{L}{2 l}-1-\frac{x}{l}\right) \text { for } x \in[L / 2-2 l, L / 2), \\
-v(x-L / 2) \text { for } x \in[L / 2, L),
\end{array}\right.
$$

with $l \leqslant L / 4$ and, of course, the periodicity condition $v(x$ $+L)=v(x)$. It is convenient to introduce the following dimensionless variables:

$$
f=F / v_{0}, \quad \alpha=l k / v_{0}, \quad \Gamma=4 l / L .
$$

In this case the function $H(x, 0 ; 0) / v_{0} \equiv T(x)$ becomes $T(x)$ $=0$ for $x \in[0, L / 2-2 l)$ and, for $x \in[L / 2-2 l, L / 2)$,

$$
\begin{aligned}
T(x)= & \left|\frac{1+f}{1-f}\right|^{\alpha} \exp \left[-\frac{4 \alpha f(1-\Gamma)}{\left(1-f^{2}\right) \Gamma}\right] \\
& \times \int_{1}^{L / 2 l-1-x / l} \operatorname{sgn}\left(f^{2}-s^{2}\right)\left|\frac{f-s}{f+s}\right|^{\alpha} d s .
\end{aligned}
$$

Explicit exact results for the probability densities and for the resultant average velocity for all values of $f$ are available and will be detailed elsewhere [9]. Here we exhibit only some of these results for the new case $0<f<1$. Figure 2 depicts a typical probability density $P(x)$ vs $x / L$ that clearly shows the agreement between the exact theoretical results and simulations. For the average velocity we find 


$$
\begin{aligned}
\langle\dot{x}\rangle= & F\left(1-\frac{\Gamma T\left(x_{1}\right)}{4 \alpha f}\left[\exp \left(\frac{4 \alpha f(1-\Gamma)}{\Gamma\left(1-f^{2}\right)}\right)-1\right]\right. \\
& +\frac{\Gamma(1-f)^{\alpha}}{2(1+f)^{\alpha}} \exp \left(\frac{4 \alpha f(1-\Gamma)}{\Gamma\left(1-f^{2}\right)}\right) \\
& \times\left\{\int_{-1}^{1} d t \frac{t\left[T[L / 2-l(t+1)]-T\left(x_{1}\right)\right]}{|t-f|^{1+\alpha}|t+f|^{1-\alpha}}\right. \\
& +\left[T\left(x_{1}\right)\left(1+\left(\frac{1+f}{1-f}\right)^{2 \alpha} \exp \left(-\frac{4 \alpha f(1-\Gamma)}{\Gamma\left(1-f^{2}\right)}\right)\right)\right. \\
& \left.\left.-T(L / 2)] \int_{-1}^{-f} d t \frac{t}{|t-f|^{1+\alpha}|t+f|^{1-\alpha}}\right\}\right)^{-1} .
\end{aligned}
$$

The above integrals can be evaluated explicitly for specific values of $\alpha$, in particular, for $\alpha=1 / 2,1$, and 2 [9]. The analytic and simulation results for the variation of the average velocity with $f$ (again with full agreement) are shown in Fig. 3. In the limit of slow switching rate, that is, in the adiabatic regime $\alpha \rightarrow 0$, one recovers the region of hypersensitive response discussed in Ref. [8], namely, $\langle\dot{x}\rangle$ $\approx 2 v_{0} \alpha / \Gamma$. The physics of this result is explained as follows. The stable and unstable fixed points of the dynamics, which coincide in the absence of forcing, are shifted apart by an amount of order of $l F / v_{0}$ for a small force. Upon each switch of the dichotomous process and for sufficiently slow switching rate, the particle will glide down to the next stable fixed point, crossing the location of the unstable fixed point of the alternate dynamics, cf. Fig. 1. As the average time between switches is $k^{-1}$, and the distance covered is $L / 2$, the mean velocity is just $\langle\dot{x}\rangle=L k / 2$, independently of $F$ and $v_{0}$. The typical time $\tau$ for a particle to escape the region around the fixed point is determined by the relation $\left(l F / v_{0}\right) \exp \left(v_{0} \tau / l\right) \approx l$. The crucial observation for hypersensitive response is that the necessary condition, $\tau \ll k^{-1}$ or $\alpha$

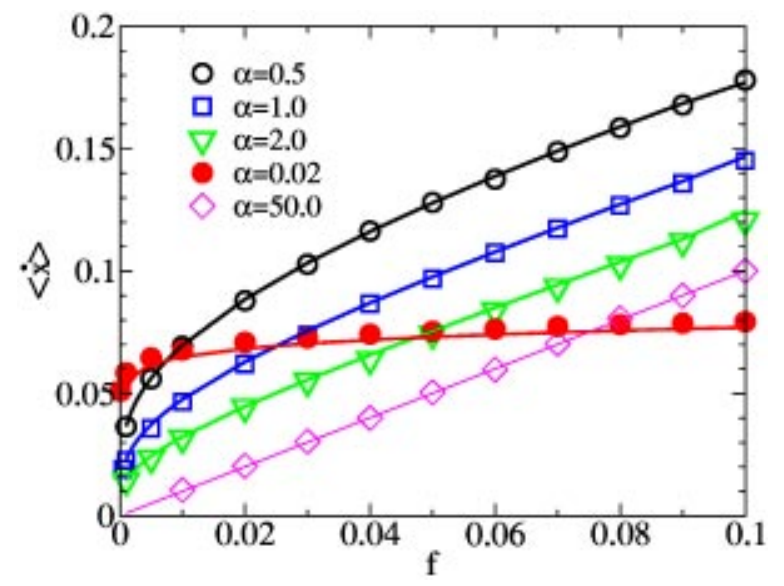

FIG. 3. Mean velocity as a function of the applied force for various values of $\alpha$. Note the hypersensitive response.

$\ll(-\ln f)^{-1}$, can be satisfied by very small forces for moderately small $\alpha$ because of the logarithmic dependence on $f$, see Fig. 3. In this figure one also observes the region of "normal" (i.e., linear) response for higher forcing or frequency and, more relevant to our preoccupation here, the strongly nonlinear dependence at very low forcing. In fact in the limit $f \rightarrow 0$, one finds from Eq. (14) that $\langle\dot{x}\rangle / v_{0}$ $\approx\left[2 \alpha \Gamma(\ln f)^{2}\right]^{-1}$. In other words, hypersensitive response is very pronounced in this region, with the velocity picking up with an infinite derivative at $f=0$.

In conclusion, the procedure presented here has resolved all technical problems related to steady state dichotomous dynamics, making possible the analytic description of cases involving the crossing of fixed points in the asymptotic regime.

This work was partially supported by the National Science Foundation under Grant Nos. PHY-9970699 and DMS0079478.
[1] N. G. Van Kampen, Stochastic Processes in Physics and Chemistry (North-Holland, Amsterdam, 1992).

[2] A. Fulinski, Phys. Rev. E 50, 2668 (1994).

[3] V.I. Klyatskin, Radiophys. Quantum Electron. 20, 382 (1977).

[4] W. Horsthemke and R. Lefever, Noise Induced Transitions (Springer-Verlag, Berlin, 1984).

[5] J.M. Sancho, Phys. Rev. A 31, 3523 (1985); J. Masoliver, K. Lindenberg, and B.J. West, ibid. 33, 2177 (1986); 34, 1481 (1986); 34, 2351 (1986); M.A. Rodriguez and L. Pesquera, ibid. 34, 4532 (1986); C.R. Doering, ibid. 35, 3166 (1987); V. Balakrishnan, C. Van den Broeck, and P. Hänggi, ibid. 38, 4213 (1988); U. Behn and K. Schiele, Z. Phys. B: Condens. Matter 77, 485 (1989); M. Kuś, E. Wajnryb, and K. Wódkiewicz, Phys. Rev. A 43, 4167 (1991); J. Olarrea, J.M.R. Parrondo, and F.J. de la Rubia, J. Stat. Phys. 79, 669 (1995); 79,
683 (1995); C. Doering, W. Horsthemke, and J. Riordan, Phys. Rev. Lett. 72, 2984 (1994).

[6] M.M. Millonas and D.R. Chialvo, Phys. Rev. E 53, 2239 (1996); V. Berdichevsky and M. Gitterman, ibid. 56, 6340 (1997); S.H. Park, S. Kim, and C.S. Ryu, Phys. Lett. A 225, 245 (1997); I. Bena, M. Copelli, and C. Van den Broeck, J. Stat. Phys. 101, 415 (2000).

[7] V. Balakrishnan, C. Van den Broeck, and I. Bena, Stochastics and Dynamics 1, 537 (2001).

[8] V. Berdichevsky and M. Gitterman, Physica A 249, 88 (1998); S.L. Ginzburg and M.A. Pustovoit, Phys. Lett. A 291, 77 (2001); Phys. Rev. Lett. 80, 4840 (1998); J. Exp. Theor. Phys. 89, 801 (1999); Eur. Phys. J. B 19, 101 (2001).

[9] I. Bena et al. (unpublished). 